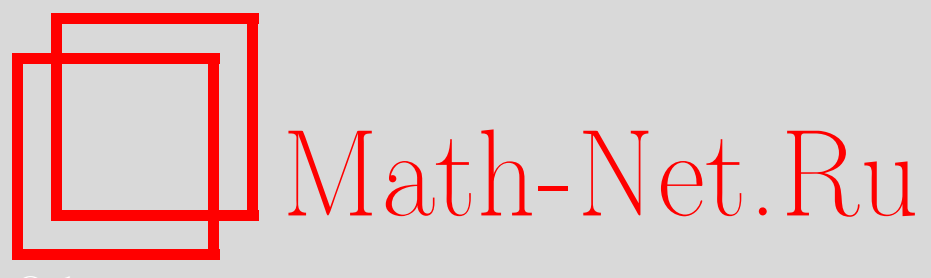

О. М. Омельян, Ю. И. Шевченко, Редукции объекта центропроективной связности и тензора аффинного кручения на распределении плоскостей, Матем. заметки, 2008, том 84, выпуск 1, 99-107

DOI: https://doi.org/10.4213/mzm5196

Использование Общероссийского математического портала Math-Net.Ru подразумевает, что вы прочитали и согласны с пользовательским соглашением http: //www . mathnet.ru/rus/agreement

Параметры загрузки:

IP: 3.85 .5 .30

26 апреля 2023 г., 12:52:48

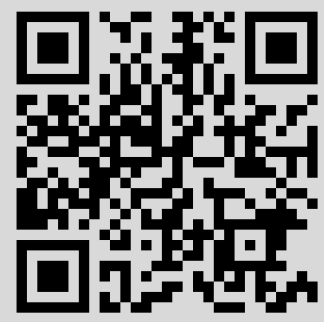


Том 84 выпуск 1 июль 2008

УДК 514.75

\title{
Редукции объекта центропроективной связности и тензора аффинного кручения на распределении плоскостей
}

\author{
О. М. Омельян, Ю. И. Шевченко
}

Проективная группа представлена в виде расслоения центропроективных реперов. В этом расслоении задана центропроективная связность, в результате чего оно стало пространством центропроективной связности. Найдены структурные уравнения этого пространства, в которые входят тензор аффинного кручения и тензор центропроективной кривизны, содержащий подтензор аффинной кривизны. Рассмотрено распределение плоскостей в проективном пространстве и ассоциированное с ним главное расслоение, имеющее 2 простейших и 2 простых в смысле работы [1] главных фактор-расслоения. В ассоциированном расслоении задана групповая связность. Показано, что объект центропроективной связности редуцируется к объекту групповой связности. Объект групповой связности содержит подобъекты плоскостной и нормальной линейных связностей, центропроективной подсвязности и аффинно-групповой связности. Определен объект кручения аффинно-групповой связности. Доказано, что он образует тензор, содержащий подтензор кручения нормальной линейной связности. Показано, что тензор аффинного кручения центропроективной связности редуцируется к тензору кручения аффинно-групповой связности.

Библиография: 11 названий.

1. Проективная группа как расслоение. Отнесем $n$-мерное проективное пространство $P_{n}$ к подвижному реперу $\left\{A, A_{I}\right\}(I, \ldots=1, \ldots, n)$ с деривационными формулами

$$
\begin{aligned}
d A & =\theta A+\omega^{I} A_{I}, \\
d A_{I} & =\theta A_{I}+\omega_{I}^{J} A_{J}+\omega_{I} A,
\end{aligned}
$$

где форма $\theta$ играет роль множителя пропорциональности, а структурные формы $\omega^{I}$, $\omega_{J}^{I}, \omega_{I}$ проективной группы $G P(n)$, эффективно действующей в пространстве $P_{n}$, удовлетворяют уравнениям (см., например, [2; с. 173])

$$
\begin{aligned}
D \omega^{I} & =\omega^{J} \wedge \omega_{J}^{I}, \\
D \omega_{J}^{I} & =\omega_{J}^{K} \wedge \omega_{K}^{I}+\delta_{J}^{I} \omega_{K} \wedge \omega^{K}+\omega_{J} \wedge \omega^{I}, \\
D \omega_{I} & =\omega_{I}^{J} \wedge \omega_{J} .
\end{aligned}
$$

Запишем структурные уравнения (1.4) в виде

$$
\begin{gathered}
D \omega_{J}^{I}=\omega_{J}^{K} \wedge \omega_{K}^{I}+\omega^{K} \wedge \omega_{J K}^{I}, \\
\omega_{J K}^{I}=-\delta_{J}^{I} \omega_{K}-\delta_{K}^{I} \omega_{J} .
\end{gathered}
$$

(С) О.М. Омельян, Ю.И. ШЕвченко, 2008 
Получили структурные уравнения (1.3), (1.6), (1.5) главного расслоения центропроективных реперов $C_{n(n+1)}\left(P_{n}\right)$, базой которого является проективное пространство $P_{n}$, а типовым слоем - центропроективная (коаффинная) группа $C_{n(n+1)}=$ $G A^{*}(n) \subset G P(n)$, действующая в любом центропроективном пространстве $P_{n}^{\circ}$ - пространстве $P_{n}$ с фиксированной точкой $A \in P_{n}$. Из формул (1.1) вытекает система уравнений стационарности точки $A$ : $\omega^{I}=0$. Эта система вполне интегрируема в силу структурных уравнений (1.3) базы $P_{n}$.

УтвержДЕнИЕ 1. Расслоение центропроективных реперов $C_{n(n+1)}\left(P_{n}\right)$ имеет главное фактор-расслоение линейных реперов $L_{n^{2}}\left(P_{n}\right)$ со структурными уравнениями (1.3), (1.6), той же базой $P_{n}$ и типовым слоем - линейной фактор-группой $L_{n^{2}}=G L(n)$, действующей в связке прямых, проходящих через биксированную точку проективного пространства $P_{n}$.

2. Центропроективная связность в проективной группе. Зададим центропроективную связность в главном расслоении $C_{n(n+1)}\left(P_{n}\right)$ приемом Лумисте [3]. Рассмотрим преобразование слоевых форм $\omega_{J}^{I}, \omega_{I}$ с помощью линейных комбинаций базисных форм $\omega^{I}$

$$
\begin{aligned}
& \widetilde{\omega}_{J}^{I}=\omega_{J}^{I}-\Pi_{J K}^{I} \omega^{K}, \\
& \widetilde{\omega}_{I}=\omega_{I}-\Pi_{I J} \omega^{J},
\end{aligned}
$$

где $\Pi_{J K}^{I}, \Pi_{I J}$ - некоторые функции, дифференциальные уравнения которых приведены ниже. Дифференцируем формы (2.1), (2.2) внешним образом:

$$
\begin{aligned}
& D \widetilde{\omega}_{J}^{I}=\omega_{J}^{M} \wedge \omega_{M}^{I}+\omega^{K} \wedge\left(d \Pi_{J K}^{I}-\Pi_{J L}^{I} \omega_{K}^{L}+\omega_{J K}^{I}\right), \\
& D \widetilde{\omega}_{I}=\omega_{I}^{L} \wedge \omega_{L}+\omega^{J} \wedge\left(d \Pi_{I J}-\Pi_{I K} \omega_{J}^{K}\right) .
\end{aligned}
$$

В первые слагаемые подставим слоевые формы, выраженные из равенств (2.1), (2.2):

$$
\begin{aligned}
D \widetilde{\omega}_{J}^{I} & =\widetilde{\omega}_{J}^{M} \wedge \widetilde{\omega}_{M}^{I}+\widetilde{\omega}_{J}^{M} \wedge \Pi_{M L}^{I} \omega^{L}+\Pi_{J K}^{M} \omega^{K} \wedge \widetilde{\omega}_{M}^{I}+\Pi_{J K}^{M} \omega^{K} \wedge \Pi_{M L}^{I} \omega^{L}+\cdots, \\
D \widetilde{\omega}_{I} & =\widetilde{\omega}_{I}^{L} \wedge \widetilde{\omega}_{L}+\widetilde{\omega}_{I}^{L} \wedge \Pi_{L K} \omega^{K}+\Pi_{I J}^{L} \omega^{J} \wedge \widetilde{\omega}_{L}+\Pi_{I J}^{L} \omega^{J} \wedge \Pi_{L K} \omega^{K}+\cdots,
\end{aligned}
$$

где многоточия обозначают соответствующие вторые слагаемые из формул (2.3). Во вторые и третьи слагаемые последних формул подставим формы связности $\widetilde{\omega}_{J}^{I}$, используя равенства (2.1), (2.2):

$$
\begin{aligned}
D \widetilde{\omega}_{J}^{I} & =\widetilde{\omega}_{J}^{K} \wedge \widetilde{\omega}_{K}^{I}+\omega^{K} \wedge\left(\Delta \Pi_{J K}^{I}+\omega_{J K}^{I}\right)-\Pi_{J K}^{M} \omega^{K} \wedge \Pi_{M L}^{I} \omega^{L}, \\
D \widetilde{\omega}_{I} & =\widetilde{\omega}_{I}^{L} \wedge \widetilde{\omega}_{L}+\omega^{J} \wedge\left(\Delta \Pi_{I J}+\Pi_{I J}^{K} \omega_{K}\right)-\Pi_{I J}^{L} \omega^{J} \wedge \Pi_{L K} \omega^{K},
\end{aligned}
$$

причем дифференциальный оператор $\Delta$ действует следующим образом:

$$
\Delta \Pi_{J K}^{I}=d \Pi_{J K}^{I}+\Pi_{J K}^{L} \omega_{L}^{I}-\Pi_{L K}^{I} \omega_{J}^{L}-\Pi_{J L}^{I} \omega_{K}^{L} .
$$

В соответствии с теоремой Картана-Лаптева [4; с. 82-83] зададим поле объекта центропроективной связности $\Pi=\left\{\Pi_{J K}^{I}, \Pi_{I J}\right\}$

$$
\begin{aligned}
\Delta \Pi_{J K}^{I}+\omega_{J K}^{I} & =\Pi_{J K L}^{I} \omega^{L}, \\
\Delta \Pi_{I J}+\Pi_{I J}^{K} \omega_{K} & =\Pi_{I J K} \omega^{K},
\end{aligned}
$$

откуда с учетом обозначения (1.7) вытекает 
УТВЕРЖДЕНИЕ 2. Объект центропроективной связности П является квазитензором, содержащим квазитензор $\Pi_{J K}^{I}$, задающий аффинную (иначе говоря, специалъную линейную) связность в расслоении линейных реперов $L_{n^{2}}\left(P_{n}\right)$.

Подставим дифференциальные уравнения (2.5), (2.6) в структурные уравнения $(2.4)$ :

$$
\begin{aligned}
D \widetilde{\omega}_{J}^{I} & =\widetilde{\omega}_{J}^{K} \wedge \widetilde{\omega}_{K}^{I}+R_{J K L}^{I} \omega^{K} \wedge \omega^{L}, \\
D \widetilde{\omega}_{I} & =\widetilde{\omega}_{I}^{L} \wedge \widetilde{\omega}_{L}+R_{I J K} \omega^{J} \wedge \omega^{K},
\end{aligned}
$$

где компоненты объекта центропроективной кривизны $R=\left\{R_{J K L}^{I}, R_{I J K}\right\}$ выражаются по формулам

$$
R_{J K L}^{I}=\Pi_{J[K L]}^{I}-\Pi_{J[K}^{M} \Pi_{|M| L]}^{I}, \quad R_{I J K}=\Pi_{I[J K]}-\Pi_{I[J}^{L} \Pi_{|L| K]} .
$$

3. Аффинное кручение. Внесем формы аффинной подсвязности (2.1) в структурные уравнения (1.3):

$$
D \omega^{I}=\omega^{J} \wedge \widetilde{\omega}_{J}^{I}+T_{J K}^{I} \omega^{J} \wedge \omega^{K}
$$

где $T_{J K}^{I}=\Pi_{[J K]}^{I}-$ компоненты объекта аффинного кручения. Из дифференциальных уравнений (2.5) с учетом обозначения (1.7) следует

$$
\Delta T_{J K}^{I} \bar{\varpi} 0
$$

где символ $\bar{\varpi}$ означает сравнение по модулю базисных форм $\omega^{I}$.

УТВЕРЖДЕНИЕ 3. Задание центропроективной связности в расслоении иентропроективных реперов $C_{n(n+1)}\left(P_{n}\right)$ превращает его в пространство центропроективной связности $C_{n(n+1), n}$ со структурными уравнениями (3.1), (2.7), (2.8), в которые входят тензор афбинного кручения $T_{J K}^{I}$ и компоненты обгекта иентропроективной кривизны $R$. Пространство $C_{n(n+1), n}$ имеет фактор-пространство афбинной связности $L_{n^{2}, n}(3.1),(2.7)$ с тензором кручения $T_{J K}^{I}$ и оббектом кривизны $R_{J K L}^{I}$.

Можно ввести [5] объект центропроективного кручения $T=\left\{T_{J K}^{I}, T_{I J}\right\}$, где $T_{I J}=$ $\Pi_{[I J]}$. Из дифференциальных уравнений (2.6) следует

$$
\Delta T_{I J}+T_{I J}^{K} \omega_{K} \bar{\varpi} 0
$$

откуда с учетом равенств (3.2) имеем

УТВЕРЖДЕНИЕ 4. Обгект центропроективного кручения Т центропроективной связности П образует тензор, содержащий подтензор аффинного кручения $T_{J K}^{I}$.

4. Центропроективная кривизна. Продолжим дифференциальные уравнения (2.5), (2.6). Сначала продифференцируем их внешним образом:

$$
\begin{aligned}
\left(\Delta \Pi_{J K L}^{I}+\Pi_{J K}^{M} \omega_{M L}^{I}-\Pi_{M K}^{I} \omega_{J L}^{M}-\Pi_{J M}^{I} \omega_{K L}^{M}\right) \wedge \omega^{L} & =0, \\
\left(\Delta \Pi_{I J K}-\Pi_{L J} \omega_{I K}^{L}-\Pi_{I L} \omega_{J K}^{L}+\Pi_{I J K}^{L} \omega_{L}\right) \wedge \omega^{K} & =0 .
\end{aligned}
$$


Разрешим квадратичные уравнения по лемме Картана, используем обозначение (1.7) для трехиндексных форм $\omega_{J K}^{I}$ и запишем результат в следующем виде:

$$
\begin{gathered}
\Delta \Pi_{J K L}^{I}-\delta_{L}^{I} \Pi_{J K}^{M} \omega_{M}+\Pi_{J K}^{I} \omega_{L}+\Pi_{L K}^{I} \omega_{J}+\Pi_{J L}^{I} \omega_{K} \bar{\varpi} 0, \\
\Delta \Pi_{I J K}+2 \Pi_{I J} \omega_{K}+\Pi_{K J} \omega_{I}+\Pi_{I K} \omega_{J}+\Pi_{I J K}^{L} \omega_{L} \bar{\varpi} 0 .
\end{gathered}
$$

Проальтернируем левые части этих равенств по двум последним индексам:

$$
\begin{gathered}
\Delta \Pi_{J[K L]}^{I}-\delta_{[L}^{I} \Pi_{|J| K]}^{M} \omega_{M}+\Pi_{[L K]}^{I} \omega_{J} \bar{\varpi} 0, \\
\Delta \Pi_{I[J K]}+\Pi_{I[J} \omega_{K]}+\Pi_{[K J]} \omega_{I}+\Pi_{I[J K]}^{L} \omega_{L} \bar{\varpi} 0 .
\end{gathered}
$$

Получим равенства для входящих в формулы (2.9) агрегатов:

$$
\begin{gathered}
\Delta \Pi_{J[K}^{M} \Pi_{|M| L]}^{I}-\delta_{[L}^{I} \Pi_{|J| K]}^{M} \omega_{M}-\Pi_{[K L]}^{I} \omega_{J} \bar{\varpi} 0, \\
\Delta \Pi_{I[J}^{L} \Pi_{|L| K]}+\Pi_{I[J}^{L} \Pi_{|L| K]}^{M} \omega_{M}-\Pi_{I[K} \omega_{J]}-\Pi_{[J K]} \omega_{I} \bar{\varpi} 0 .
\end{gathered}
$$

Вычитаем левые части этих равенств из соответствующих частей равенств (4.1) и используем обозначения (2.9):

$$
\Delta R_{J K L}^{I} \bar{\varpi} 0, \quad \Delta R_{I J K}+R_{I J K}^{L} \omega_{L} \bar{\varpi} 0 .
$$

УТВЕРЖДЕНИЕ 5. Обгект иентропроективной кривизны $R$ образует тензор, содержащий подтензор аффинной кривизны $R_{J K L}^{I}$.

5. Распределение плоскостей. В проективном пространстве $P_{n}$ рассмотрим распределение $m$-мерных плоскостей, которое представим как $n$-параметрическое семейство $S_{n}$ центрированных $m$-плоскостей $P_{m}^{\circ}, 0<m<n$. Произведем разбиение значений индексов

$$
I=\{i, \alpha\}: \quad i, \ldots=1, \ldots, m, \quad \alpha, \ldots=m+1, \ldots, n .
$$

Осуществим специализацию подвижного репера $\left\{A, A_{i}, A_{\alpha}\right\}$, помещая вершины $A$, $A_{i}$ на плоскость $P_{m}^{\circ}$, причем точку $A-$ в ее центр. Из деривационных формул $(1.2)$ имеем

$$
d A_{i}=\theta A_{i}+\omega_{i}^{j} A_{j}+\omega_{i}^{\alpha} A_{\alpha}+\omega_{i} A .
$$

Формулы (1.1), (5.1) дают уравнения стационарности центрированной плоскости $P_{m}^{\circ}$ : $\omega^{I}=0, \omega_{i}^{\alpha}=0$. Выбирая $n$ форм $\omega^{I}$ в качестве базисных, запишем уравнения распределения $S_{n}$ в виде

$$
\omega_{i}^{\alpha}=\Lambda_{i J}^{\alpha} \omega^{J}
$$

Дифференцируем эти уравнения:

$$
\left(\Delta \Lambda_{i J}^{\alpha}-\delta_{J}^{\alpha} \omega_{i}\right) \wedge \omega^{J}=0,
$$

где $\delta_{J}^{\alpha}$ - обобщенный символ Кронекера,

$$
\Delta \Lambda_{i J}^{\alpha}=d \Lambda_{i J}^{\alpha}+\Lambda_{i J}^{\beta} \omega_{\beta}^{\alpha}-\Lambda_{j J}^{\alpha} \omega_{i}^{j}-\Lambda_{i K}^{\alpha} \omega_{J}^{K} .
$$

Разрешим квадратичные уравнения (5.3) по лемме Картана

$$
\Delta \Lambda_{i J}^{\alpha}-\delta_{J}^{\alpha} \omega_{i}=\Lambda_{i J K}^{\alpha} \omega^{K} \quad\left(\Lambda_{i[J K]}^{\alpha}=0\right) .
$$

Запишем дифференциальные уравнения (5.4) подробнее

$$
\Delta \Lambda_{i j}^{\alpha}=\left(\Lambda_{i j K}^{\alpha}+\Lambda_{i \beta}^{\alpha} \Lambda_{j K}^{\beta}\right) \omega^{K}, \quad \Delta \Lambda_{i \beta}^{\alpha}-\Lambda_{i j}^{\alpha} \omega_{\beta}^{j}-\delta_{\beta}^{\alpha} \omega_{i}=\Lambda_{i \beta K}^{\alpha} \omega^{K} .
$$


УТВЕРЖДЕНИЕ 6. Фундаментальный объект 1-го порядка $\Lambda=\left\{\Lambda_{i j}^{\alpha}, \Lambda_{i \beta}^{\alpha}\right\}$ распределения $S_{n}$ является квазитензором, содержсащим подтензор $\Lambda_{i j}^{\alpha}$.

Тензор $\Lambda_{i j}^{\alpha}$ порождает тензор неголономности $N_{i j}^{\alpha}=\Lambda_{[i j]}^{\alpha}$ распределения $S_{n}$. Из структурных уравнений (1.3) с учетом уравнений (5.2) следует

$$
D \omega^{\alpha}=\omega^{\beta} \wedge\left(\omega_{\beta}^{\alpha}-\Lambda_{i \beta}^{\alpha} \omega^{i}\right)+N_{i j}^{\alpha} \omega^{i} \wedge \omega^{j} .
$$

Если тензор неголономности обращается в нуль: $N_{i j}^{\alpha}=0$, то распределение $S_{n}$ превращается в голономное распределение $S_{n}^{\prime}$, которое представляет собой [6] расслоение пространства $P_{n}$ на $(n-m)$-мерное многообразие $m$-поверхностей $S_{m}$, рассматриваемых как семейства центрированных касательных плоскостей $P_{m}^{\circ}$. Структурные уравнения (5.5) принимают вид

$$
D \omega^{\alpha}=\omega^{\beta} \wedge\left(\omega_{\beta}^{\alpha}-\Lambda_{i \beta}^{\alpha} \omega^{i}\right)
$$

Система уравнений $\omega^{\alpha}=0$ становится вполне интегрируемой. Она выделяет из голономного распределения $S_{n}^{\prime}$ поверхность $S_{m}$ с уравнениями $\omega_{i}^{\alpha}=\Lambda_{i j}^{\alpha} \omega^{j}$. Более сильное условие $\Lambda_{i j}^{\alpha}=0$ влечет вырождение поверхности $S_{m}$ в плоскость $P_{m}^{\circ}$.

6. Ассоциированное расслоение и его фактор-расслоения. Внешние дифференциалы вторичных форм $\omega_{j}^{i}, \omega_{i}, \omega_{\beta}^{\alpha}, \omega_{\alpha}^{i}, \omega_{\alpha}$ имеют вид

$$
\begin{aligned}
D \omega_{j}^{i} & =\omega_{j}^{k} \wedge \omega_{k}^{i}+\omega^{K} \wedge \omega_{j K}^{i}, \\
D \omega_{i} & =\omega_{i}^{j} \wedge \omega_{j}+\omega^{J} \wedge \omega_{i J}, \\
D \omega_{\beta}^{\alpha} & =\omega_{\beta}^{\gamma} \wedge \omega_{\gamma}^{\alpha}+\omega^{I} \wedge \omega_{\beta I}^{\alpha}, \\
D \omega_{\alpha}^{i} & =\omega_{\alpha}^{j} \wedge \omega_{j}^{i}+\omega_{\alpha}^{\beta} \wedge \omega_{\beta}^{i}+\omega^{J} \wedge \omega_{\alpha J}^{i}, \\
D \omega_{\alpha} & =\omega_{\alpha}^{i} \wedge \omega_{i}+\omega_{\alpha}^{\beta} \wedge \omega_{\beta},
\end{aligned}
$$

где

$$
\begin{aligned}
& \omega_{j K}^{i}=\Lambda_{j K}^{\alpha} \omega_{\alpha}^{i}-\delta_{j}^{i} \omega_{K}-\delta_{K}^{i} \omega_{j}, \quad \omega_{i J}=\Lambda_{i J}^{\alpha} \omega_{\alpha}, \\
& \omega_{\beta I}^{\alpha}=-\Lambda_{i I}^{\alpha} \omega_{\beta}^{i}-\delta_{\beta}^{\alpha} \omega_{I}-\delta_{I}^{\alpha} \omega_{\beta}, \quad \omega_{\alpha J}^{i}=-\delta_{J}^{i} \omega_{\alpha} .
\end{aligned}
$$

Получили структурные уравнения (1.3), (6.1)-(6.5) главного расслоения $G_{r}\left(P_{n}\right)$, базой которого является проективное пространство $P_{n}$, а типовым слоем - подгруппа $G_{r} \subset C_{n(n+1)}$ стационарности центрированной плоскости $P_{m}^{\circ}$, причем $r=$ $n(n-m)+m^{2}+n$. Расслоение $G_{r}\left(P_{n}\right)$, ассоциированное с распределением $S_{n}$, имеет четыре главных факторрасслоения [7] над той же базой $P_{n}$ со следующими структурными уравнениями:

- $(1.3),(6.1)$ - расслоение плоскостных линейных реперов $L_{m^{2}}\left(P_{n}\right)$ с типовым слоем $L_{m^{2}}=G L(m)$ - линейной факторгруппой, действующей в связке прямых плоскости $P_{m}^{\circ}$ с центром $A$;

- $(1.3),(6.3)$ - расслоение нормальных линейных реперов $\mathscr{L}_{(n-m)^{2}}\left(P_{n}\right)$ с типовым слоем $\mathscr{L}_{(n-m)^{2}}=G L(n-m)$ - линейной факторгруппой, действующей в проективном факторпространстве $\mathrm{P}_{n-m-1}=P_{n} / P_{m}^{\circ}$;

- $(1.3),(6.1),(6.2)$ - расслоение центропроективных реперов $C_{m(m+1)}\left(P_{n}\right)$ с типовым слоем $C_{m(m+1)}=G A^{*}(m)$ - центропроективной (коаффинной) факторгруппой, действующей в центрированной плоскости $P_{m}^{\circ}$; 
- $(1.3),(6.1),(6.3),(6.4)$ - аффинное ассоциированное расслоение $H_{\bar{r}}\left(P_{n}\right)$ с типовым слоем $H_{\bar{r}}, \bar{r}=n(n-m)+m^{2},-$ аффинной факторгруппой [8] группы $G_{r} \subset G P(n)$, действующей в связке прямых пространства $P_{n}$ с центром $A$, в которой выделяется связка прямых плоскости $P_{m}^{\circ}$ с тем же центром $A$.

Теорема 1. Распределение $S_{n}$ плоскостей $P_{m}^{\circ}$ в проективном пространстве $P_{n}$ редуцирует расслоение центропроективных реперов $C_{n(n+1)}\left(P_{n}\right)$ до главного расслоения $G_{r}\left(P_{n}\right)$, которое имеет два простейших [1] факторрасслоения линейных реперов $L_{m^{2}}\left(P_{n}\right), \mathscr{L}_{(n-m)^{2}}\left(P_{n}\right)$ и два простых [1] бакторрасслоения $C_{m(m+1)}\left(P_{n}\right)$, $H_{\bar{r}}\left(P_{n}\right)$.

7. Ассоциированное пространство групповой связности. Зададим групповую связность в главном расслоении $G_{r}\left(P_{n}\right)$ приемом Лумисте с помощью форм связности

$$
\begin{gathered}
\widehat{\omega}_{j}^{i}=\omega_{j}^{i}-\Gamma_{j K}^{i} \omega^{K}, \quad \widehat{\omega}_{i}=\omega_{i}-\Gamma_{i J} \omega^{J}, \quad \widehat{\omega}_{\beta}^{\alpha}=\omega_{\beta}^{\alpha}-\Gamma_{\beta I}^{\alpha} \omega^{I}, \\
\widehat{\omega}_{\alpha}^{i}=\omega_{\alpha}^{i}-\Gamma_{\alpha J}^{i} \omega^{J}, \quad \widehat{\omega}_{\alpha}=\omega_{\alpha}-\Gamma_{\alpha I} \omega^{I},
\end{gathered}
$$

причем компоненты объекта групповой связности $\Gamma=\left\{\Gamma_{j K}^{i}, \Gamma_{i J}, \Gamma_{\beta I}^{\alpha}, \Gamma_{\alpha J}^{i}, \Gamma_{\alpha I}\right\}$ удовлетворяют следующим дифференциальным уравнениям [7]:

$$
\begin{aligned}
\Delta \Gamma_{j K}^{i}+\omega_{j K}^{i} & =\Gamma_{j K L}^{i} \omega^{L}, \\
\Delta \Gamma_{i J}+\Gamma_{i J}^{k} \omega_{k}+\omega_{i J} & =\Gamma_{i J K} \omega^{K}, \\
\Delta \Gamma_{\beta I}^{\alpha}+\omega_{\beta I}^{\alpha} & =\Gamma_{\beta I J}^{\alpha} \omega^{J}, \\
\Delta \Gamma_{\alpha J}^{i}-\Gamma_{j J}^{i} \omega_{\alpha}^{j}+\Gamma_{\alpha J}^{\beta} \omega_{\beta}^{i}+\omega_{\alpha J}^{i} & =\Gamma_{\alpha J K}^{i} \omega^{K}, \\
\Delta \Gamma_{\alpha I}-\Gamma_{i I} \omega_{\alpha}^{i}+\Gamma_{\alpha I}^{i} \omega_{i}+\Gamma_{\alpha I}^{\beta} \omega_{\beta} & =\Gamma_{\alpha I J} \omega^{J} .
\end{aligned}
$$

Отсюда с учетом обозначений (6.6) вытекает [9]

Теорема 2. Объект групповой связности Г содержит два простейших [1] подобгекта: объект плоскостной линейной связности $\Gamma_{1}=\left\{\Gamma_{j K}^{i}\right\}$, обгект нормальной линейной связности $\Gamma_{2}=\left\{\Gamma_{\beta I}^{\alpha}\right\}$ и два простых [1] подобгекта: обгект центропроективной связности $\Gamma_{3}=\left\{\Gamma_{1}, \Gamma_{i J}\right\}$, обгект афбинно-групповой связности $\Gamma_{4}=\left\{\Gamma_{1}, \Gamma_{2}, \Gamma_{\alpha J}^{i}\right\}$, задающие связности в соответствующих факторрасслоениях $L_{m^{2}}\left(P_{n}\right), \mathscr{L}_{(n-m)^{2}}\left(P_{n}\right), C_{m(m+1)}\left(P_{n}\right), H_{\bar{r}}\left(P_{n}\right)$. Обгект $\Gamma$ образует геометрический обгект (точнее, квазитензор) лишь в совокупности с фундаментальным квазитензором $\Lambda$. Квазитензор $\{\Gamma, \Lambda\}$ содержит четыре простых подквазитензора $\left\{\Gamma_{1}, \Lambda\right\},\left\{\Gamma_{2}, \Lambda\right\},\left\{\Gamma_{3}, \Lambda\right\},\left\{\Gamma_{4}, \Lambda\right\}$.

Дифференциальные уравнения (7.2)-(7.6) компонент объекта связности Г определяют структурные уравнения форм групповой связности (7.1):

$$
\begin{aligned}
D \widehat{\omega}_{j}^{i} & =\widehat{\omega}_{j}^{k} \wedge \widehat{\omega}_{k}^{i}+K_{j K L}^{i} \omega^{K} \wedge \omega^{L}, \\
D \widehat{\omega}_{i} & =\widehat{\omega}_{i}^{j} \wedge \widehat{\omega}_{j}-+K_{i J K} \omega^{J} \wedge \omega^{K}, \\
D \widehat{\omega}_{\beta}^{\alpha} & =\widehat{\omega}_{\beta}^{\gamma} \wedge \widehat{\omega}_{\gamma}^{\alpha}+K_{\beta I J}^{\alpha} \omega^{I} \wedge \omega^{J}, \\
D \widehat{\omega}_{\alpha}^{i} & =\widehat{\omega}_{\alpha}^{j} \wedge \widehat{\omega}_{j}^{i}+\widehat{\omega}_{\alpha}^{\beta} \wedge \widehat{\omega}_{\beta}^{i}+K_{\alpha J K}^{i} \omega^{J} \wedge \omega^{K}, \\
D \widehat{\omega}_{\alpha} & =\widehat{\omega}_{\alpha}^{i} \wedge \widehat{\omega}_{i}+\widehat{\omega}_{\alpha}^{\beta} \wedge \widehat{\omega}_{\beta}+K_{\alpha I J} \omega^{I} \wedge \omega^{J},
\end{aligned}
$$


где компоненты объекта групповой кривизны $K=\left\{K_{j K L}^{i}, K_{i J K}, K_{\beta I J}^{\alpha}, K_{\alpha J K}^{i}, K_{\alpha I J}\right\}$ выражаются по формулам

$$
\begin{gathered}
K_{j K L}^{i}=\Gamma_{j[K L]}^{i}-\Gamma_{j[K}^{k} \Gamma_{|k| L]}^{i}, \quad K_{i J K}=\Gamma_{i[J K]}-\Gamma_{i[J}^{j} \Gamma_{|j| K]}, \\
K_{\beta I J}^{\alpha}=\Gamma_{\beta[I J]}^{\alpha}-\Gamma_{\beta[I}^{\gamma} \Gamma_{|\gamma| J]}^{\alpha}, \\
K_{\alpha J K}^{i}=\Gamma_{\alpha[J K]}^{i}-\Gamma_{\alpha[J}^{j} \Gamma_{|j| K]}^{i}-\Gamma_{\alpha[J}^{\beta} \Gamma_{|\beta| K]}^{i}, \quad K_{\alpha I J}=\Gamma_{\alpha[I J]}-\Gamma_{\alpha[I}^{i} \Gamma_{|i| J]}-\Gamma_{\alpha[I}^{\beta} \Gamma_{|\beta| J]} .
\end{gathered}
$$

ТЕОРема 3. Задание групповой связности Г в ассоицированном расслоении $G_{r}\left(P_{n}\right)$ превращает его в пространство групповой связности $G_{r, n}$ со структурными уравнениями (1.3), (7.7)-(7.11), которое имеет два простейших [1] бакторпространства линейной связности $L_{m^{2}, n}(1.3),(7.7), \mathscr{L}_{(n-m)^{2}, n}(1.3),(7.9)$ и два простых [1] факторпространства со связностями: пространство центропроективной (коаффинной) связности $C_{m(m+1), n}(1.3),(7.7),(7.8)$ и пространство аффинно-групповой связности $H_{\bar{r}, n}(1.3),(7.7),(7.9),(7.10)$.

8. Редукция объекта центропроективной связности. Представим дифференциальные уравнения (2.5), (2.6) компонент объекта центропроективной связности П в более подробном виде, соответствующем компонентам объекта групповой связности $Г$, в котором учтем уравнения (5.2) распределения $S_{n}$ :

$$
\begin{aligned}
\Delta \Pi_{j K}^{i}+\Pi_{j K}^{\alpha} \omega_{\alpha}^{i}-\delta_{j}^{i} \omega_{K}-\delta_{K}^{i} \omega_{j} & =\left(\Pi_{j K L}^{i}+\Pi_{\alpha K}^{i} \Lambda_{j L}^{\alpha}\right) \omega^{L}, \\
\Delta \Pi_{\beta K}^{\alpha}-\Pi_{i K}^{\alpha} \omega_{\beta}^{i}-\delta_{\beta}^{\alpha} \omega_{K}-\delta_{K}^{\alpha} \omega_{\beta} & =\left(\Pi_{\beta K L}^{\alpha}-\Pi_{\beta K}^{i} \Lambda_{i L}^{\alpha}\right) \omega^{L}, \\
\Delta \Pi_{i K}^{\alpha}-\delta_{K}^{\alpha} \omega_{i} & =\left(\Pi_{i K L}^{\alpha}-\Pi_{i K}^{k} \Lambda_{k L}^{\alpha}+\Pi_{\beta K}^{\alpha} \Lambda_{i L}^{\beta}\right) \omega^{L}, \\
\Delta \Pi_{\alpha K}^{i}+\Pi_{\alpha K}^{\beta} \omega_{\beta}^{i}-\Pi_{j K}^{i} \omega_{\alpha}^{j}-\delta_{K}^{i} \omega_{\alpha} & =\Pi_{\alpha K L}^{i} \omega^{L} \\
\Delta \Pi_{i J}+\Pi_{i J}^{k} \omega_{k}+\Pi_{i J}^{\alpha} \omega_{\alpha} & =\left(\Pi_{i J K}+\Pi_{\alpha J} \Lambda_{i K}^{\alpha}\right) \omega^{K}, \\
\Delta \Pi_{\alpha J}-\Pi_{i J} \omega_{\alpha}^{i}+\Pi_{\alpha J}^{i} \omega_{i}+\Pi_{\alpha J}^{\beta} \omega_{\beta} & =\Pi_{\alpha J K} \omega^{K} .
\end{aligned}
$$

Сопоставляя дифференциальные уравнения (5.4) и (8.3), положим $\Pi_{i K}^{\alpha}=\Lambda_{i K}^{\alpha}$, т.е. охватим часть компонент объекта центропроективной связности П в случае данного распределения $S_{n}$. Тогда объект П сокращается до объекта $\bar{\Pi}=\left\{\Pi_{j K}^{i}, \Pi_{\beta K}^{\alpha}, \Pi_{\alpha K}^{i}, \Pi_{i J}\right.$, $\left.\Pi_{\alpha J}\right\}$, который можно отождествить с объектом групповой связности $\Gamma$, что следует из сопоставления уравнений $(8.1),(8.2),(8.4)-(8.6)$ и (7.2)-(7.6) с учетом обозначений (6.6).

ТЕОРема 4. Если в проективном пространстве $P_{n}$ дано распределение плоскостей $S_{n}$, то объект центропроективной связности П, задающий связность в проективной группе $G P(n)$, представленной как расслоение чентропроективных реперов $C_{n(n+1)}\left(P_{n}\right)$, редуцируется $к$ обгекту групповой связности $\Gamma$, задающему связность в главном расслоении $G_{r}\left(P_{n}\right)$, ассочиированном с распределением $S_{n}$.

9. Кручение аффинно-групповой связности. Запишем структурные уравнения (1.3) проективного пространства $P_{n}$ в подробном виде:

$$
\begin{aligned}
D \omega^{i} & =\omega^{j} \wedge \omega_{j}^{i}+\omega^{\alpha} \wedge \omega_{\alpha}^{i}, \\
D \omega^{\alpha} & =\omega^{i} \wedge \omega_{i}^{\alpha}+\omega^{\beta} \wedge \omega_{\beta}^{\alpha} .
\end{aligned}
$$


Подставим в структурные уравнения (9.2) уравнения (5.2) распределения $S_{n}$, но преобразуем их с помощью обобщенного символа Кронекера к другому по сравнению с (5.5) виду

$$
D \omega^{\alpha}=\omega^{\beta} \wedge \omega_{\beta}^{\alpha}+\delta_{I}^{i} \omega^{I} \wedge \Lambda_{i J}^{\alpha} \omega^{J} .
$$

Внесем формы $\widehat{\omega}_{j}^{i}, \widehat{\omega}_{\alpha}^{i}, \widehat{\omega}_{\beta}^{\alpha}$ аффинно-групповой связности $\Gamma_{4}$ в структурные уравнения (9.1), (9.3):

$$
D \omega^{i}=\omega^{j} \wedge \widehat{\omega}_{j}^{i}+\omega^{\alpha} \wedge \widehat{\omega}_{\alpha}^{i}+S_{J K}^{i} \omega^{J} \wedge \omega^{K}, \quad D \omega^{\alpha}=\omega^{\beta} \wedge \widehat{\omega}_{\beta}^{\alpha}+S_{I J}^{\alpha} \omega^{I} \wedge \omega^{J},
$$

где компоненты объекта $S=\left\{S_{J K}^{i}, S_{I J}^{\alpha}\right\}$, который назовем объектом кручения аффинно-групповой связности, выражаются по формулам

$$
\begin{gathered}
S_{J K}^{i}=\delta_{[J}^{j} \Gamma_{|j| K]}^{i}+\delta_{[J}^{\alpha} \Gamma_{|\alpha| K]}^{i}, \\
S_{I J}^{\alpha}=\delta_{[I}^{\beta} \Gamma_{|\beta| J]}^{\alpha}+\delta_{[I}^{i} \Lambda_{|i| J]}^{\alpha} .
\end{gathered}
$$

Найдем дифференциальные уравнения для обобщенных символов Кронекера $\delta_{J}^{i}, \delta_{J}^{\alpha}$, исходя из уравнений для обычного символа Кронекера $\delta_{J}^{I}$

$$
\Delta \delta_{J}^{I}=0,
$$

которые проверяются непосредственно:

$$
\Delta \delta_{J}^{I}=d \delta_{J}^{I}+\delta_{J}^{K} \omega_{K}^{I}-\delta_{K}^{I} \omega_{J}^{K}=0+\omega_{J}^{I}-\omega_{J}^{I}=0 .
$$

Из уравнений (9.7) имеем

$$
\begin{aligned}
& \Delta \delta_{J}^{i}+\delta_{J}^{\alpha} \omega_{\alpha}^{i}=0, \\
& \Delta \delta_{J}^{\alpha}+\delta_{J}^{i} \omega_{i}^{\alpha}=0 .
\end{aligned}
$$

С учетом уравнений (5.2) уравнения (9.9) запишем короче

$$
\Delta \delta_{J}^{\alpha} \bar{\varpi} 0 .
$$

Уравнения (5.4), (7.2), (7.4), (7.5), (9.8), (9.10) с помощью выражений трехиндексных форм из (6.6) позволяют найти сравнения для компонент (9.5), (9.6)

$$
\begin{gathered}
\Delta S_{J K}^{i}+\delta_{[J}^{j} \Lambda_{|j| K]}^{\alpha} \omega_{\alpha}^{i}-\delta_{[J}^{i} \omega_{K]}-\left(\delta_{[J}^{j} \omega_{|j|}+\delta_{[J}^{\alpha} \omega_{|\alpha|}\right) \delta_{K]}^{i}+\delta_{[J}^{\beta} \Gamma_{|\beta| K]}^{\alpha} \omega_{\alpha}^{i} \bar{\varpi} 0, \\
\Delta S_{I J}^{\alpha}-\delta_{[I}^{\alpha} \omega_{J]}-\left(\delta_{[I}^{\beta} \omega_{|\beta|}+\delta_{[I}^{i} \omega_{|i|}\right) \delta_{J]}^{\alpha} \bar{\varpi} 0 .
\end{gathered}
$$

Слагаемые в круглых скобках запишем с помощью обычного символа Кронекера, приведем подобные члены и воспользуемся формулой (9.6)

$$
\Delta S_{J K}^{i}+S_{J K}^{\alpha} \omega_{\alpha}^{i} \bar{\varpi} 0, \quad \Delta S_{I J}^{\alpha} \bar{\varpi} 0 .
$$

Теорема 5. Обгект кручения $S$ аффинно-групповой связности $\Gamma_{4}$ образует тензор, содержащий подтензор $S_{I J}^{\alpha}-$ тензор кручения нормальной линейной связносmu $\Gamma_{2} \subset \Gamma_{4}$.

Теорема 6. Распределение $S_{n}$ в проективном пространстве $P_{n}$ позволяет редуиировать тензор афбинного кручения $T_{J K}^{I}$ иентропроективной связности П $к$ тензору кручения $S$ афбинно-групповой связности $\Gamma_{4}$.

Действительно, запишем равенства (3.2) подробнее с учетом уравнений (5.2):

$$
\Delta T_{J K}^{i}+T_{J K}^{\alpha} \omega_{\alpha}^{i} \bar{\varpi} 0, \quad \Delta T_{J K}^{\alpha} \bar{\varpi} 0,
$$

что совпадает с равенствами (9.11). 
Вывод. В качестве структурных уравнений базы пространства групповой связности $G_{r, n}$ и его факторпространства аффинно-групповой связности $H_{\bar{r}, n}$ лучше использовать не уравнения (1.3) для проективного пространства $P_{n}$, а уравнения (9.4), в которых учтены [10] уравнения (5.2) распределения $S_{n}$. Тогда структурные уравнения пространств групповой связности $G_{r, n}(9.4),(7.7)-(7.11)$ и аффинно-групповой связности $H_{\bar{r}, n}(9.4),(7.7),(7.9),(7.10)$ замкнуты в смысле Лаптева [11], т.е. в их правых частях стоят внешние произведения тех форм, от которых в левых частях берутся внешние дифференциалы.

\section{СПИСОК ЦИТИРОВАННОЙ ЛИТЕРАТУРЫ}

[1] Ю. И. Шевченко, Оснащения центропроективных многообразий, Изд-во КГУ, Калининград, 2000.

[2] Ш. Кобаяси, Группь преобразований в дифференииальной геометрии, Наука, М., 1986.

[3] Ю.И. Шевченко, "Приемы Лаптева и Лумисте задания связности в главном расслоении", Дифференциальная геометрия многообразий фигур, 37, Изд-во КГУ, Калининград, 2006, 179-187.

[4] Л. Е. Евтушик, Ю. Г. Лумисте, Н. М. Остиану, А. П. Широков, “Дифференциально-геометрические структуры на многообразиях", Итоги науки и техники. Проблемы геометрии, 9, ВИНИТИ, М., 1979, 5-247.

[5] В. Г. Лемлейн, "Локальные центропроективные пространства и связности в дифференцируемом многообразии", Литовский матем. сб., 4, № 1, 1964, 41-132.

[6] Г.Ф. Лаптев, Н. М. Остиану, "Распределения $m$-мерных линейных элементов в пространстве проективной связности. I", Тр. геом. семин., 3, ВИНИТИ, М., 1971, 49-94.

[7] О.М. Омельян, "Нетензорность объекта кривизны групповой связности на распределении плоскостей", Дифференциальная геометрия многообразий фигур, $\mathbf{3 3}$, Изд-во КГУ, Калининград, 2002, 74-78.

[8] Ю.И. Шевченко, "Аффинная, коаффинная и линейная факторгруппы в подгруппе проективной группы”, Проблемы математических и физических наук, Изд-во КГУ, Калининград, 2002, 38-39.

[9] О.М. Омельян, "Об объекте кривизны групповой связности на распределении плоскостей”, Тр. матем. центра им. Н. И. Лобачевского, 18, Изд-во Казанск. матем. об-ва, Казань, 2002, 69.

[10] O. M. Omelyan, The Object of Curvature of the Distributed Linear Connection, Programme \& Abstracts of Fourth European Congress of Mathematics, Royal Institute of Technology, Stockholm, 2004.

[11] Г. Ф. Лаптев, “Дифференциальная геометрия погруженных многообразий”, Tp. MMO, 2 (1953), 275-382.

\section{О. М. Омельян}

Поступило

Российский государственный университет им. И. Канта,

26.03.2007

г. Калининград

Исправленный вариант

E-mail: olga_omelyan2002@mail.ru

24.12.2007

\section{Ю. И. Шевченко}

Российский государственный университет им. И. Канта

E-mail: geometry@albertina.ru 